\title{
EDITORIAL
}

\section{Oncology trials-the elephant in the room}

E ach year, when new annual cancer incidence and mortality statistics are published, the media are quick and happy to report that cancer mortality is reducing, despite some increases in incidence. There is a lot for the oncology community to celebrate and be proud of in these declining mortality rates and the decades of work that have produced them. However, late-phase clinical trials for anticancer drugs have the highest failure rates of all the clinical areas. ${ }^{1}$ Furthermore, those drugs that are successful often do not provide sufficient improvement on already available therapies, meaning that health-care providers often cannot afford to make them available to the patients they were designed for. ${ }^{2}$ Against this backdrop, it is time to discuss oncology clinical trials-the elephant in the room.

Perhaps it is misleading to call these trials the elephant in the room, as it is not as if the oncology community is unaware of the problem. Nevertheless, changes in how clinical trials are conducted have been remarkably slow in coming (with some notable exceptions-I-SPY and BATTLE being the best examples). Although we all agree that the current situation is far from optimal and that 'something should be done,' there is considerably less consensus on what that something should be. Frankly, given that many cancer trials take an average of 800 days to start, ${ }^{3}$ the system needs a radical overhaul. What we have witnessed recently reflects more of a tinkering.

As a journal, we feel that part of our responsibility to the oncology community is to shine some light on important issues. To that end, we have commissioned a focus issue on the theme of clinical trials. We asked clinicians, a statistician, drug developers and those involved in making regulatory decisions to write about how they view the situation and to put forward areas that are ripe for improvement. As part of our research into preparing this focus issue, inevitably we have also come to some conclusions, which we would like to share here. We recognize that we do not have definitive answers for such a complex and important challenge, but our comments are intended to spark thought and provide some suggestions.

The changing needs of regulatory bodies is one such challenging area. For example, historically, chemotherapy drugs were assessed in combination in the clinic for efficacy without the necessity for each of those drugs to already show efficacy as a monotherapy. ${ }^{4}$ This type of trial is now very rarely, if ever, undertaken. However, without this type of trial it would not have been possible for such advances as the identification of the curative MOPP (nitrogen mustard, vincristine, procarbazine and prednisone) regimen for patients with lymphoma. ${ }^{4}$ Indeed,

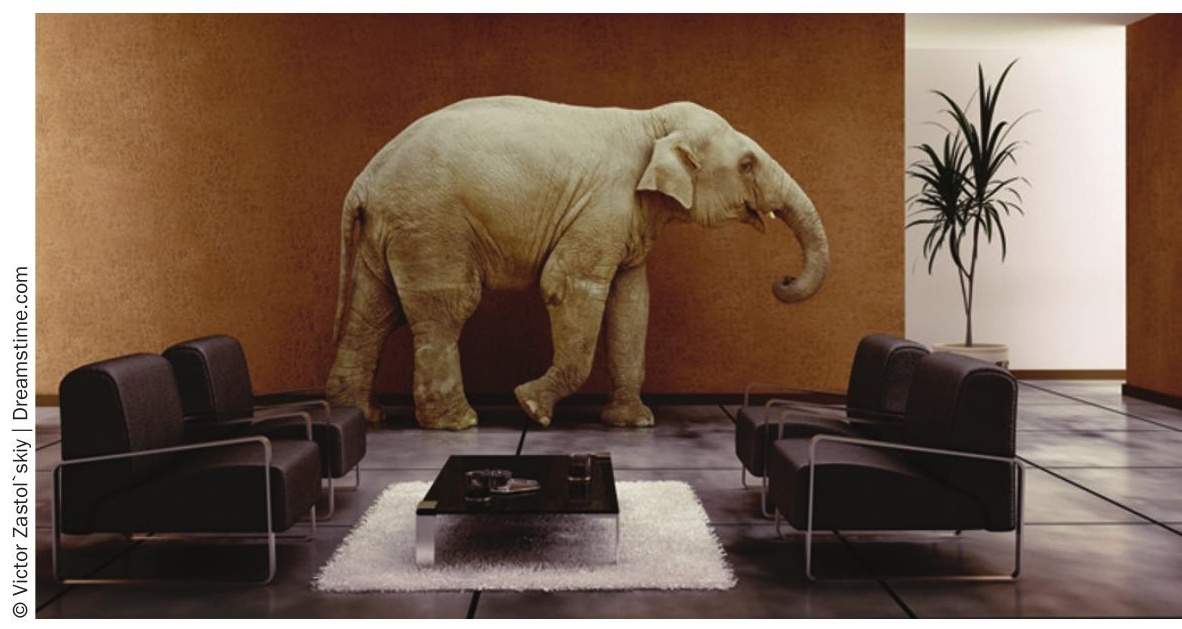

when we were speaking with Vincent DeVita about the issue of oncology clinical trials, he commented that if he were starting the research that confirmed the efficacy of MOPP today, he would not be able to undertake the critical trials. Obviously, we are not advocating a position in which any researcher can undertake any clinical trial that they wish. But, perhaps it should be possible that with sufficient and robust preclinical data, and with critical safety data for the compounds, clinical trials in which a combination therapy is the starting point should be a possibility. This would also avoid the situation where phase III trials are initiated because previous trials did not include additional relevant combination arms as comparators, an approach that would not only save time and unnecessary patient accural but also money.

An obvious hurdle-in addition to the complexity of clinical trial design and recruitment-is the man-made barrier of the restrictions imposed by regulators. For example, most institutional review boards will not approve a trial concept or initiation unless the study has FDA or NCI approval. Clinicians do not have the freedom of being able to rapidly adjust trial protocols as they did in the past, which has stifled some of the progress in the field.

One area, however, in which changes should be possible is that of preclinical or 'phase 0' trials that offer a great deal in terms of improving the success rates of later trial stages. ${ }^{5,6}$ After the initial identification of a suitable molecular anticancer target or promising chemotherapeutic drug, the time spent at the preclinical validation stage is critical to ensure that the right molecular entities are taken forward into patients. Unfortunately, this is an area where there are many challenges to overcome, not least the simple fact that the current system financially
Rebecca Kirk is an Associate Editor and Lisa Hutchinson is the Chief Editor of Nature Oncology.

Competing interests The authors declare no competing interests. Reviews Clinical 
4 ...our

comments are

intended to

spark thought

and provide ... suggestions 77 rewards those who are fastest to market and not necessarily those who are the most careful and diligent in their preclinical assessments. ${ }^{7}$ A suggestion to overcome this is to 'stop the clock' on patents to allow the expensive and time-consuming task of careful preclinical assessment and biomarker discovery to be completed, without detriment to the potential financial rewards to be had from a launched product. ${ }^{7}$ Unfortunately, this novel suggestion would likely be too open to abuse to be feasible, but the idea is a starting point that might lead to successful changes in regulation.

We also want to discuss patient recruitment to clinical trials. As agents are increasingly developed to target specific markers, it is more likely that patients will require prescreening for biomarkers prior to enrollment in a clinical trial. However, who should pay for the assessment? If this prescreening is not needed by the patient for their standard treatment then it is unlikely to be paid for by their usual health-care provider; but if the assessment is not done, then the patient cannot be recruited to the trialsomething of a Catch-22. In France, an initiative has been set up to perform centralized prescreening of this kind for approved anticancer therapies that are associated with a biomarker. ${ }^{8}$ This assessment is intended to allow patients access to the correct targeted anticancer therapy. It is easy to see how an initiative of this type could be expanded to assess patients for eligibility for enrollment in clinical trials; however, that is not part of the focus at present.

A suggestion to improve recruitment is the possibility of the disclosure in the public domain of those centers with a poor recruitment record. ${ }^{9}$ The idea that a center's recruitment target, actual accrual and reasons for any disparity should be made public will probably not be popular. However, these data would be informative on several levels. It would be possible to determine if there are trends in recruitment difficulties and if they can be rectified going forward, which in our opinion would be the main benefit of this suggestion. It might also be of interest in terms of trial subgroup analyses and patient location.

Another crucial issue is asking the right trial question and choosing the correct end points for the study. Progression-free survival is a common primary end point and recent investigational therapies often improve this end point by only a few months. Although these gains can be of clinical significance to patients, health-care providers who make assessments based on cost-effectiveness frequently do not approve such agents for use or reimbursement. The result: the intended patient populations do not get access to these expensive, but efficacious, drugs. ${ }^{2}$ Perhaps earlier liaison with funding bodies to assess the relative cost-effectiveness of efficacy of these agents in parallel with development might help to avoid this situation, which is destined to increase dramatically in light of the extremely crowded and cost prohibitive drug marketplace.

Close collaboration between industry partners to avoid redundancy in developing multiple agents with the same target would be a considerable step forward, as highlighted by the recent Merck and AstraZeneca collaboration for their respective AKT and MEK inhibitors. Discontinuation of agents earlier in the drug development process that do not meet the criteria suggested by Sharma and Schilsky, ${ }^{10}$ would also help to reduce failure rates. As stated earlier, if more recognition was given to rewarding careful and thorough preclinical progress rather than first-to-market drug development, drug companies might also be more willing to work together to invest in preclinical research. Such collaborative efforts might also help validate the robustness of academic research findings. As $50 \%$ of published preclinical results are not reproducible by industry, steps to improve such reproducibility with a new public-funded access scheme to leverage academic and industry-reported efforts could help future clinical trial design and limit development of agents with a poor target definition.

Obviously, we have cherry picked just a few examples to discuss here and have not even begun to scratch the surface in terms of what is possible and what has been proposed within the community. It is our hope that this editorial and the articles in this clinical trials focus issue will be illuminating and of interest to our readers, and that possibly they might contribute in a small way to the ongoing changes in clinical trial practice that will benefit researchers, clinicians and patients alike.

doi:10.1038/nrclinonc.2012.33
1. Arrowsmith, J. Trial watch: Phase III and submission failures: 2007-2010. Nat. Rev. Drug Discov. 10, 87 (2011).

2. Rinaldi, F., George, E. \& Adler, A. I. NICE guidance on cetuximab, bevacizumab, and panitumumab for treatment of metastatic colorectal cancer after first-line chemotherapy. Lancet Oncol. 13, 233-234 (2012).

3. DeVita, V.T. Jr The clinical trials system is broken. Nat. Clin. Pract. Oncol. 5, 683 (2008).

4. Chabner, B. A. \& Roberts T. G. Jr. Timeline: Chemotherapy and the war on cancer. Nat. Rev. Cancer 5, 65-72 (2005).
5. Yap, T. A., Sandhu, S. K., Workman, P. \& de Bono, J. S. Opinion: Envisioning the future of early anticancer drug development. Nat. Rev. Cancer 10, 514-523 (2010).

6. Rubin, E. H. \& Gilliland, D. G. Drug development and clinical trials-the path to an approved cancer drug. Nat. Rev. Clin. Oncol. 9, 215-222 (2012).

7. Jonsson, B. \& Bergh, J. Opinion: Hurdles in anticancer drug development from a regulatory perspective. Nat. Rev. Clin. Oncol. 9, 236-243 (2012).

8. Institut National du Cancer. Les plateformes hospitalières de génétique moléculaire [online,
French], http://www.e-cancer.fr/soins/platesformes-hospitalieres-de-genetique-moleculaire (2012).

9. Dal-Ré, R. et al. (2011) Disclosure of investigators' recruitment performance in multicenter clinical trials: a further step for research transparency. PLoS Med. 8, e1001149 (2011).

10. Sharma, M. R. \& Schilsky, R. L. Role of randomized phase III trials in an era of effective targeted therapies. Nat. Rev. Clin. Oncol. 9, 208-214 (2012). 